\title{
High Energy Emission from Supernova Remnants
}

\author{
Jacco Vink ${ }^{1}$ \\ ${ }^{1}$ Astronomical Institute, University of Utrecht, PO Box 80000, NL-3508TA,Utrecht, \\ The Netherlands \\ j.vink@astro.uu.nl
}

\begin{abstract}
This paper discusses several aspects of current research on high energy emission from supernova remnants, covering the following main topics: 1) The recent evidence for magnetic field amplification near supernova remnant shocks, which makes that cosmic rays are more efficiently accelerated than previously thought. 2) The evidence that ions and electrons in some remnants have very different temperatures, and only equilibrate through Coulomb interactions. 3) The evidence that the explosion that created Cas A was asymmetric, and seems to have involved a jet/counter jet structure. And finally, 4), I will argue that the unremarkable properties of supernova remnants associated with magnetar candidates, suggest that magnetars are not formed from rapidly $(P \approx 1 \mathrm{~ms})$ rotating proto-neutron stars. It is therefore more likely that they are formed from massive progenitor stars with high magnetic fields.
\end{abstract}

Keywords. (ISM:) supernova remnants, (ISM:) cosmic rays, X-rays: ISM.

\section{Introduction}

Supernova remnants (SNRs) are important high energy sources. Not only are they thought to be the major source of Galactic cosmic rays of energies up to at least $10^{15} \mathrm{eV}$, they are, until a supernova occurs in the Galaxy, also the only Galactic sources with which can get a direct view on supernova explosions and nucleosynthesis.

Moreover, SNRs are sources in which interesting physical processes take place, some of which can now be spatially or spectroscopically resolved with the current generation of high spatial and spectral resolution X-ray telescopes, Chandra, and XMM-Newton.

\section{Collisionless shocks}

That the hot plasma of SNRs that we observe with X-ray telescopes exists is somewhat of a surprise. The reason is that SNR shocks take place in the tenuous interstellar medium, with typical densities of $n \sim 1 \mathrm{~cm}^{-3}$. Two body atomic collisions in such a medium are so rare that, were it not for the generation of plasma waves, plasmas could move through each other without hardly any noticeable interaction. Because of this lack of two body collisions (Coulomb interactions) SNR shocks are called collisionless.

The small collision rates in SNRs has two consequences. One of them, non-equilibrium ionization (NEI), has been well established since the eighties (see e.g. Liedahl 1999, for an introduction). SNR plasmas are usually in NEI, meaning that the plasma has not yet had time to reach ionization equilibrium. In other words the plasma is still ionizing. NEI plasmas are usually characterized by the parameter $n_{\mathrm{e}} t$, i.e. the product of electron density and time. Plasmas with temperatures of $k T_{\mathrm{e}} \sim 1 \mathrm{keV}$ are out of equilibrium when $n_{\mathrm{e}} t \lesssim 10^{12} \mathrm{~cm}^{-3} \mathrm{~s}$.

Less well known is that there is considerable uncertainty how collisionless plasmas are heated by shocks, and in particular whether this leads immediately downstream of the 

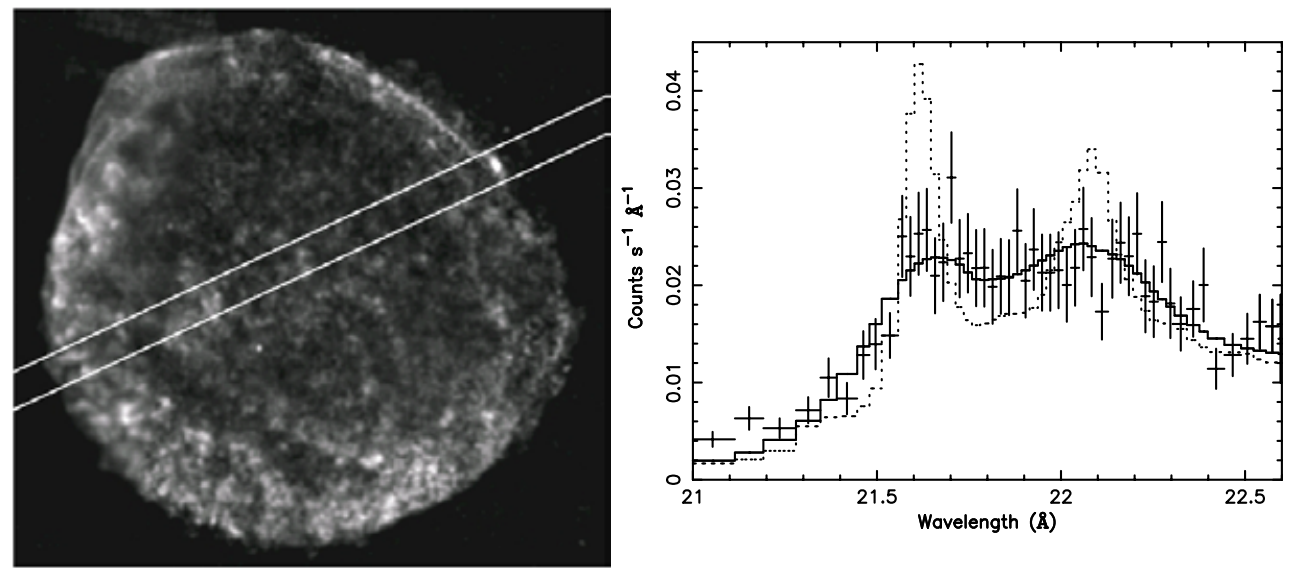

Figure 1. On the left: Map of OVII emission of SN1006 made from several Chandra-ACIS observations. The lines indicate the region observed by the $X M M$-Newton RGS instrument. The target was the bright knot in the northwest. Right: Detail of the RGS1 spectrum of the northwestern knot, showing O VII He $\alpha$ line emission. The dashed line is the best fit model without line broadening, whereas the solid line shows the model including thermal line broadening (Vink et al. 2003).

shock to equal temperatures for all particles (electrons, ions) involved. Basic considerations, such as conservation of mass, momentum and energy, lead to the Rankine-Hugoniot relations, but it is not clear whether collisionless shock heating leads to equal temperatures for all particles, or whether it heats different plasma elements to different temperatures. In the latter case one expects that the temperature of each species is proportional to $k T_{i} \propto m_{i} v_{s}^{2}$, with $m_{i}$ the particle mass and $v_{s}$ the shock speed. Far downstream of the shock the particles will eventually equilibrate their energies. Also the Coulomb equilibration of electrons and ions is governed by the parameter $n_{\mathrm{e}} t$. Equilibration is reached when $n_{\mathrm{e}} t \lesssim 10^{12} \mathrm{~cm}^{-3} \mathrm{~s}$.

Over the last decade several observations in the optical (Ghavamian et al. 2001, 2003), UV (Raymond et al. 1995; Laming et al. 1996; Korreck et al. 2004), and X-rays (Vink et al. 2003) have been used to address this issue. They all rely on the fact that ion temperatures can be measured by observing thermal Doppler broadenings. For SNRs this can only be done for the limb brightened edges of the shells, because far inside the shell, bulk motions in the line of sight dominate the line broadening.

In Fig. 1 X-ray evidence is shown that for SN 1006 the ion temperature is much hotter, $k T_{\text {OVII }} \sim 500 \mathrm{keV}$, than the electron temperature $k T_{\mathrm{e}} \approx 1.5 \mathrm{keV}$. Of course what is normally measured is the electron temperature, as this determines the X-ray continuum shape and emission line ratios.

SN1006 is a very suitable target for such a study, as its plasma is very far out of equilibrium, $\log n_{\mathrm{e}} t=9.5$. However, optical, $\mathrm{H} \alpha$, measurements of line broadening do not rely on low $n_{\mathrm{e}} t$ values, as $\mathrm{H} \alpha$ emission only occurs very close to the shock front. Using H $\alpha$ measurements of several SNRs, Rakowski et al. (2003) have shown that slow equilibration of temperatures is probably a function of shock velocity or Mach number, as only the fastest shocks appear to have substantial differences between electron and proton temperatures.

\section{Cosmic ray acceleration and magnetic field amplification}

One of the important findings of Chandra concerning SNRs was that with its CCD detectors it was able to pick out thin, non-thermal X-ray emitting filaments (Hwang et al. 

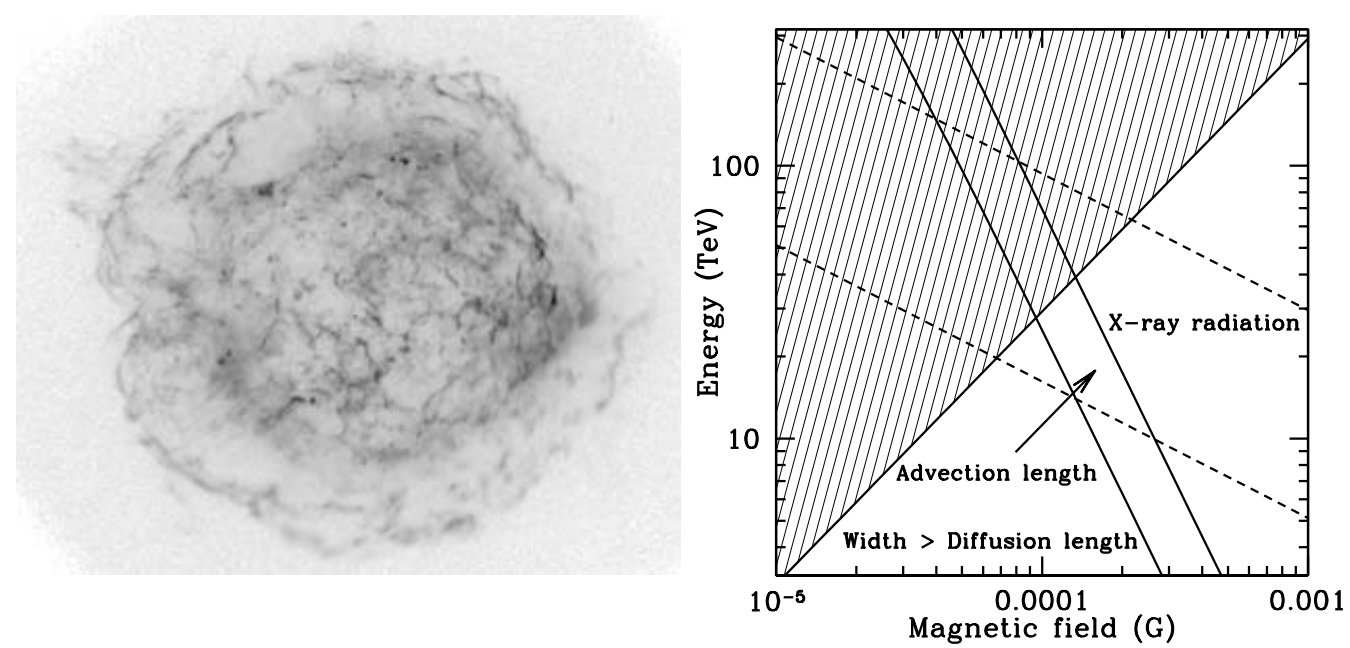

Figure 2. A deep Chandra image of Cas A (Hwang et al. 2004) in the 4-6 keV continuum band (left). Note the thin filaments, marking the border of the remnant (NB the point spread function is not uniform). The remnant has a radius of about $2.5^{\prime}$. Right: Determination of the maximum electron energy versus magnetic field strength for the region just downstream of Cas A's shock front, as determined from the thickness of the filaments. The shaded area is excluded, because the filament width cannot be smaller than the minumum possible diffusion length (c.f. Vink \& Laming 2003).

2002; Gotthelf et al. 2001). Vink \& Laming (2003) showed that these filaments probably emit synchrotron emission, with electron energies $\gtrsim 10 \mathrm{TeV}$. The narrow widths of these filaments are then best interpreted as the result of synchrotron losses.

The reason is that the plasma downstream of the shock sweeps the relativistic particles away from the shock. At the same time the electrons rapidly lose energy, so that at some point away from the shock front the electrons only emit synchrotron radiation at energies below the X-ray band. For standard high Mach number shocks the plasma velocity with respect to the shock front is given by $\Delta v=\frac{1}{4} v_{s}$. So for the width of the filaments one can write $\Delta r=\frac{1}{4} v_{s} \tau_{\text {loss }}$, with the synchrotron loss time given by $\tau_{\text {loss }}=635 /\left(B^{2} E\right)$. In order to disentangle the electron energy $E$, and the average downstream magnetic field strength $B$ one has to use the fact that the peak photon energy as a result of synchrotron radiation is $\epsilon=7.4 E^{2} B \mathrm{keV}$. Fig. 2 shows graphically what for Cas $\mathrm{A}$ the possible values for $B$ and $E$ are. It turns out that the magnetic field is high $B=200-500 \mu \mathrm{G}$ for Cas A, but also for other young SNRs (e.g. Bamba et al. 2005; Ballet 2005), which is much stronger than might be expected if the magnetic field is just the shock compressed mean Galactic field.

This may be surprising, but it is a nice confirmation of recent theoretical work that indicates that strong cosmic ray streaming close to fast SNR shocks may lead to nonlinear amplification of magnetic fields (Bell \& Lucek 2001; Bell 2004).

In fact this solves a piece of the puzzle concerning cosmic ray acceleration. SNRs were for a long time thought to be the most plausible sources of Cosmic Rays up to or beyond $3 \times 10^{15} \mathrm{eV}$, at which energy the cosmic ray spectrum has a break. However, with only mean Galactic magnetic field values SNRs are not able to efficiently accelerate particles up to even $10^{14} \mathrm{eV}$ (Lagage \& Cesarsky 1983). It looks now that Chandra has solved this problem. 

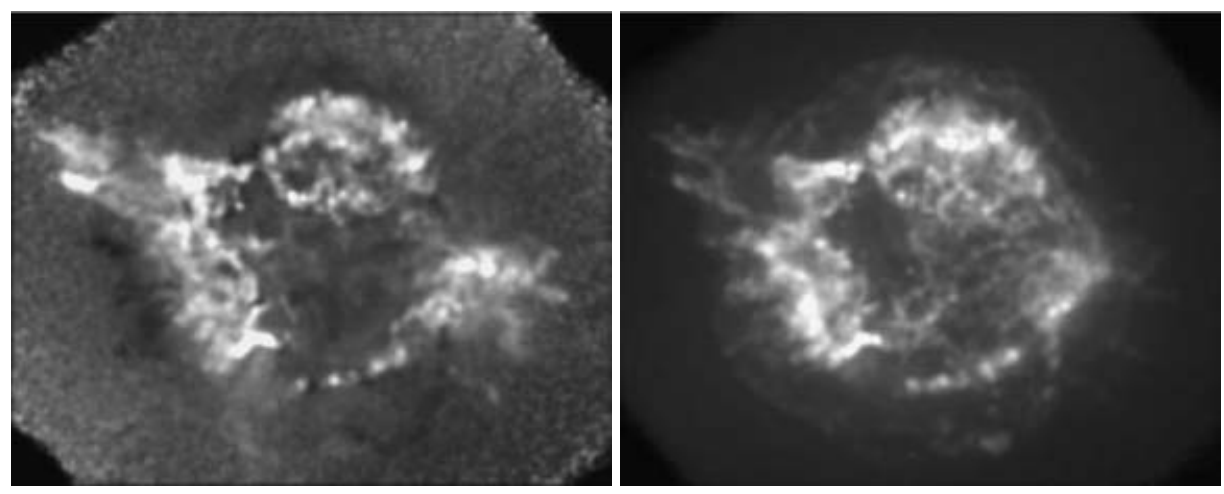

Figure 3. Left: Image based on the deep Chandra observation of Cas A, which has been processed to bring out the jet/counter jet structure (Vink 2004a; Hwang et al. 2004). Right: For comparison a Chandra X-ray image taken in the band of Si XIII line emission.

Although X-ray observations show that one necessary ingredient, large, turbulent magnetic fields are present (see also Vink 2004b), there is still no direct evidence that SNRs accelerate also ions up to high energies. Note that ions are the main ingredient of the cosmic rays that bombard the earth atmosphere. In that respect the many SNRs that have been observed with Cherenkov telescopes, in particular H.E.S.S. (Aharonian et al. 2004), are very promising. The reason is that collisions of relativistic ions result in the production of pions, and $\pi^{0}$ particles decay into two photons, giving rise to $\gamma$-ray emission. However, also inverse Compton scattering of background photons by relativistic electrons produces $\gamma$-ray emission. In that case X-ray and Cherenkov $\gamma$-ray telescopes may observe the same electron cosmic ray population. It has not yet been resolved which mechanism is responsible for the $\gamma$-ray emission from SNRs.

\section{Asymmetric supernova explosions: a link with GRBs?}

The origin of cosmic rays may be an almost century old problem, but an equally fascinating, but more recent problem is the nature of gamma-ray bursts (GRBs). It is becoming more and more clear that long duration GRBs are probably associated with core collapse supernovae of subclass Type Ibc (Stanek et al. 2003). However, the mechanism that generates the powerful relativistic jets that we observe as GRBs is not well known. The collapsar model (MacFadyen et al. 2001) is one of the most popular models. In this model the stellar core collapses into a black hole that accretes matter, and generates jets. An alternative model is magneto-rotational jet formation (Akiyama et al. 2003).

In this light it is interesting that it was recently discovered that the bright Galactic SNR Cas A seems to have exploded with a jet/counter jet. A normal X-ray image of Cas A does not immediately reveal this, but dividing a narrow band image dominated by Si XIII line emission by a narrow band image with Mg XI line emission brings out a clear jet/counter jet structure (Fig. 3, Vink 2004a; Hwang et al. 2004). The spectra of the jet reveal an apparent absence of $\mathrm{Ne}$ and $\mathrm{Mg}$. The dominant elements seem to be $\mathrm{Si}$, $\mathrm{S}$, and Ar, but some Fe seems also present. The emission measure of the jet combined with the average velocity of the plasma suggest quite a high kinetic energy in the jet, $\sim 5 \times 10^{50} \mathrm{erg}$, about $25 \%$ of the total explosion energy.

So in terms of the total explosion energy the jets seem to contain a substantial, but not a dominant fraction of the energy. The jets seem not to have been relativistic jets, like those 

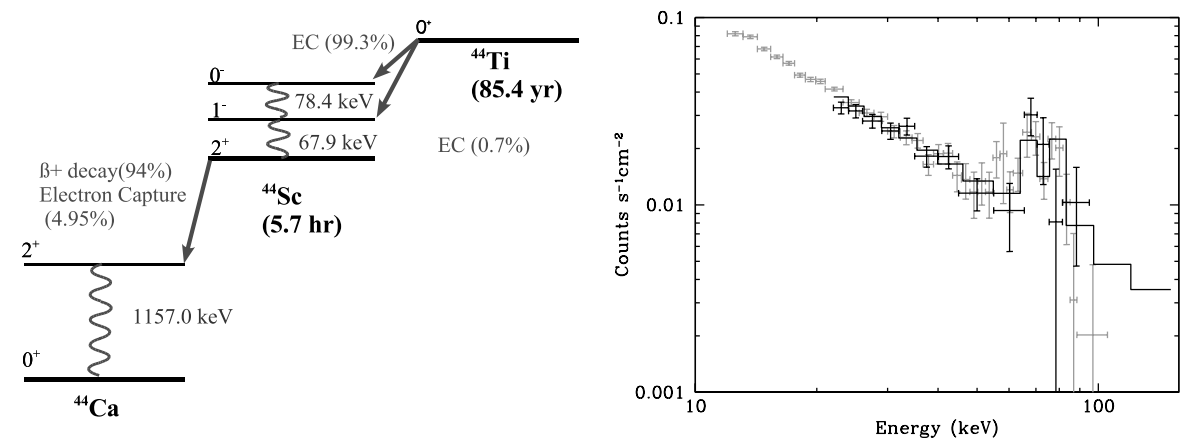

Figure 4. Left: The radio active decay scheme of ${ }^{44} \mathrm{Ti}$. Right: hard X-ray spectrum of Cas A, as observed by BeppoSAX (in gray) and INTEGRAL, both showing clear signs of emission around $68 \mathrm{keV}$ and $78 \mathrm{keV}$ due to ${ }^{44} \mathrm{Ti}$ decay lines (Vink 2005).

of GRBs. Nevertheless, perhaps the same underlying mechanism produces both types of jets. In that respect it is interesting that Cas A is likely the result of a Type Ib explosion, i.e. it belonged to the same supernova subclass with which GRBs are associated. However, it is unlikely that the collapsar model in its present form is responsible for the jets in Cas A, because there is the simple fact that the explosion appears to have resulted in the formation of a recently detected neutron star (Tananbaum 1999) rather than a black hole.

Interestingly, not only the presence of a jet/counter jet makes Cas A an interesting SNR from the point of view of the explosion mechanism. Equally interesting is that Fe-rich knots seem to have been ejected with greater speed than the Si-rich material synthesized further away from the core. This is clear from the presence of Fe-rich knots in the southeast of the remnant, outside of the main Si-rich shell (Hughes et al. 2000; Hwang \& Laming 2003).

In the north the Fe is projected to the inside of the Si-rich shell. However, this appears to be a projection effect, because the measured Doppler velocities of Fe in the north are higher than for Si (Willingale et al. 2002). It is not clear how much of the Fe in Cas A is still unshocked, but some of the shocked Fe must have been ejected with velocities of up to $7800 \mathrm{~km} \mathrm{~s}^{-1}$. There is no obvious symmetry to the Fe-rich ejecta, so their emergence is probably related to hydrodynamical instabilities close to the core of the explosion (Kifonidis et al. 2003).

The 3D morphology of Cas A as reconstructed from the Doppler imaging obtained from $X M M-N e w t o n$ gives further evidence that the explosion was deviating from spherical (Willingale et al. 2002). Apart from the jets and Fe-rich knots, the ejecta can best be described by a donut shape. This morphology also appears to describe best the high resolution spectroscopy data of the Small Magellanic Cloud remnant 1E0102.2-7219, obtained by the grating spectrometers of Chandra (Flanagan et al. 2004).

There is one other observational fact concerning Cas A that may point to an intrinsically asymmetric explosion: the presence of ${ }^{44} \mathrm{Ti}$, which suggest a high initial ${ }^{44} \mathrm{Ti}$ yield of $\sim 10^{-4} M_{\odot} \cdot{ }^{44} \mathrm{Ti}$ is exclusively an explosive nucleosynthesis product, and is synthesized close to the core of the explosion. It is an alpha-rich freeze out product (Arnett 1996), and as such very sensitive to explosion energy and explosion asymmetries.

The decay of ${ }^{44} \mathrm{Ti}(\tau=86 \mathrm{yr})$ is accompanied by three strong $\gamma$-ray lines (Fig. 4), which have been detected by CGRO-COMPTEL (the $1157 \mathrm{keV}$ line, Iyudin et al. 1994), and BeppoSAX (the 68 and $78 \mathrm{keV}$ lines, Vink et al. 2001), with lines fluxes of $\sim 2 \times$ $10^{-5} \mathrm{ph} \mathrm{cm}^{-2} \mathrm{~s}^{-1}$. The fact that the line emission is caused by radio-active decay makes 

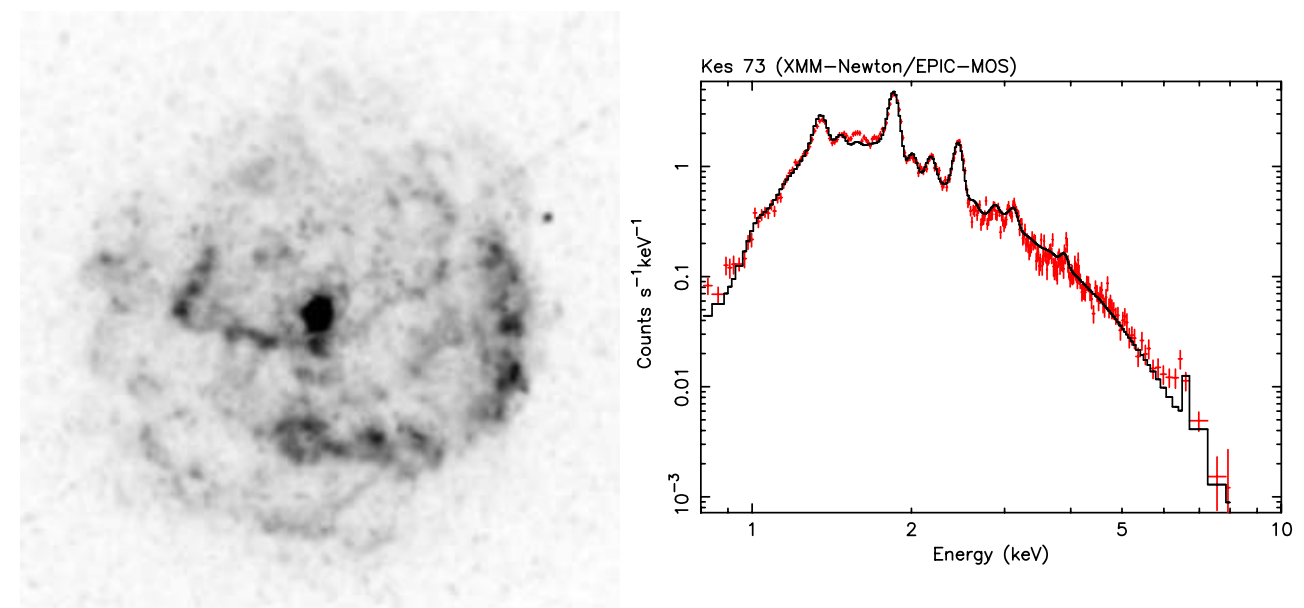

Figure 5. Left: Chandra image of Kes 73. The AXP itself is highly saturated and shows up as a dark spot in the center of the remnant. Right: XMM-Newton EPIC-MOS spectrum of Kes 73, with the best fit Sedov model shown as a solid line. From this Sedov model (Borkowski et al. 2001) one can estimate the total kinetic energy of the supernova (Vink et al. in preparation).

that with ${ }^{44} \mathrm{Ti}$ we can also probe the unshocked ${ }^{44} \mathrm{Ti}$ ejecta. Cas $\mathrm{A}$ is therefore a target of ESA's $\gamma$-ray observatory INTEGRAL. Preliminary results of the observations have been published in Vink (2005) (Fig. 4).

\section{The remarkable magnetars and the unremarkable supernova remnants in which they reside}

Core collapse supernovae obtain their energy from the gravitational potential energy released when the core of a massive star collapses. The classical view is that core collapse would either result in the creation of a radio pulsar with typical magnetic fields of $B_{d i p} \sim$ $10^{12} \mathrm{G}$, or otherwise in the formation of a black hole. However, over the last decade it has become clear that some neutron stars appear to have very high magnetic fields, up to $B_{\text {dip }} \sim 10^{15} \mathrm{G}$. Such neutron stars are called "magnetars". It is thought that two classes of X-ray pulsars are manifestations of magnetars. Depending on the presence or absence of (soft) $\gamma$-ray flashes, these pulsars are either labeled Soft-gamma-ray-repeaters (SGRs), or Anomalous X-ray Pulsars (AXPs) (see Kaspi \& Gavriil 2004; Woods \& Thompson 2004, for reviews).

It is not clear what causes the creation of a magnetar, but two mechanisms have been proposed. Perhaps the most popular explanation is that magnetars are created during the core collapse of massive stars with a high angular momentum. This results in the formation of a proto-neutron star which rotates non-uniformly with an average rotation period close to the break up limit $P \sim 1 \mathrm{~ms}$. This allows for the efficient operation of an $\alpha-\Omega$ dynamo, which rapidly amplifies the magnetic field (Duncan \& Thompson 1992). Once the high magnetic field is in place, a magnetar will lose most of its angular momentum due to magnetic breaking in less than a few hunderd seconds, i.e. during the supernova explosion itself (e.g. Thompson et al. 2004). This means that most of the rotational energy will be rapidly pumped into the supernova ejecta. A neutron star with $P=1 \mathrm{~ms}$ has a rotational energy of $\sim 3 \times 10^{52} \mathrm{erg}$, which should be compared to an average core collapse supernova energy of $\sim 10^{51} \mathrm{erg}$.

What interests us here is the fact that of the dozen or so magnetar candidates, three are associated with bright SNRs: Kes 73, N49, and CTB 109. So one would expect that 
the kinetic energy of these SNRs is exceptionally high, as the ejecta have been energized by the rapid spin down of the rapidly rotating magnetar. However, the kinetic energies of these SNRs are unremarkable: A study of the energetics with XMM-Newton-EPIC spectroscopy reveals that the kinetic energies of Kes 73, N49, and CTB 109 are resp. $0.8 \times 10^{51} \mathrm{erg}, 2.0 \times 10^{51} \mathrm{erg}$, and $0.7 \times 10^{51} \mathrm{erg}$ (Vink et al. 2005 , in preparation, and Sasaki et al. 2004). This is far short of the $\sim 3 \times 10^{52}$ erg expected if magnetars owed their existence to the operation of an $\alpha-\Omega$ dynamo (Duncan \& Thompson 1992). In fact one can put a lower limit on the initial spin period, by equating the observed SNR energies to the initial rotational energy of the pulsar. This gives $\left.P_{i}>5.6 \sqrt{(} E_{S N R} / 10^{51} \mathrm{erg}\right) \mathrm{ms}$, which is closer to the classical initial spin period of radio pulsars, $10 \mathrm{~ms}$, than to the break up limit of a neutron star.

So the unremarkable energies of SNRs associated with magnetars imply that magnetars are the result of collapses of the cores of massive stars with high magnetic fields (see also Ferrario \& Wickramasinghe 2005), rather than from stars with a high angular momentum.

\section{Summary}

I have shown that X-ray studies of SNRs provides us with important information on collisionless shock physics, cosmic ray acceleration and magnetic field amplification by SNRs.

Moreover, by studying SNRs we can learn about the details of the supernova explosions that caused them. For example, the kinematics and spatial distribution of metals in Cas A reveal that the explosion was intrinsically asymmetric, and was accompanied by the emergence of a jet/counter jet system. And I have discussed that the most remarkable property of SNRs associated with magnetars is that they are unremarkable: Their energies are similar to those of other SNRs, which suggests that magnetars were not formed from proto-neutron stars with period close to $1 \mathrm{~ms}$.

\section{References}

Aharonian, F. A. et al. 2004, Nat, 432, 75

Akiyama, S., Wheeler, J. C., Meier, D. L. \& Lichtenstadt, I. 2003, ApJ, 584, 954

Arnett, D. 1996, Supernovae and Nucleosynthesis (NJ: Princeton Univ. Press)

Ballet, J. 2005, astro-ph/0503309

Bamba, A., Yamazaki, R., Yoshida, T., Terasawa, T. \& Koyama, K. 2005, ApJ, 621, 793

Bell, A. R. 2004, MNRAS, 353, 550

Bell, A. R. \& Lucek, S. G. 2001, MNRAS, 321, 433

Borkowski, K. J., Lyerly, W. J. \& Reynolds, S. P. 2001, ApJ, 548, 820

Duncan, R. C. \& Thompson, C. 1992, ApJ, 392, L9

Ferrario, L. \& Wickramasinghe, D. T. 2005, MNRAS, 356, 615

Flanagan, K. A., Canizares, C. R., Dewey, D., et al. 2004, ApJ, 605, 230

Ghavamian, P., Rakowski, C. E., Hughes, J. P. \& Williams, T. B. 2003, ApJ, 590, 833

Ghavamian, P., Raymond, J., Smith, R. C. \& Hartigan, P. 2001, ApJ, 547, 995

Gotthelf, E. V. et al. 2001, ApJ, 552, L39

Hughes, J. P., Rakowski, C. E., Burrows, D. N. \& Slane, P. O. 2000, ApJ, 528, L109

Hwang, U., Decourchelle, A., Holt, S. S. \& Petre, R. 2002, ApJ, 581, 1101

Hwang, U. \& Laming, J. M. 2003, ApJ, 597, 362

Hwang, U. et al. 2004, ApJ, 000, 000

Iyudin, A. F. et al. 1994, A\&A, 284, L1

Kaspi, V. M. \& Gavriil, F. P. 2004, Nuclear Physics B Proceedings Supplements, 132, 456

Kifonidis, K., Plewa, T., Janka, H.-T. \& Müller, E. 2003, A\&A, 408, 621

Korreck, K. E., Raymond, J. C., Zurbuchen, T. H. \& Ghavamian, P. 2004, ApJ, 615, 280 
Lagage, P. O. \& Cesarsky, C. J. 1983, A\&A, 125, 249

Laming, J. M., Raymond, J. C., McLaughlin, B. M. \& Blair, W. P. 1996, ApJ, 472, 267

Liedahl, D. A. 1999, Lecture Notes in Physics, Berlin Springer Verlag, 520, 189

MacFadyen, A. I., Woosley, S. E. \& Heger, A. 2001, ApJ, 550, 410

Rakowski, C. E., Ghavamian, P. \& Hughes, J. P. 2003, ApJ, 590, 846

Raymond, J. C., Blair, W. P. \& Long, K. S. 1995, ApJ, 454, L31

Sasaki, M., Plucinsky, P. P., Gaetz, T. J., et al. 2004, ApJ, 617, 322

Stanek, K. Z. et al. 2003, ApJ, 591, L17

Tananbaum, H. 1999, IAU circ., 7246, 1

Thompson, T. A., Chang, P. \& Quataert, E. 2004, ApJ, 611, 380

Vink, J. 2004a, New Astronomy Review, 48, 61

Vink, J. 2004b, astro-ph/0409517

Vink, J. 2005, Adv. Space Res., 35, 976

Vink, J. \& Laming, J. M. 2003, ApJ, 584, 758

Vink, J., Laming, J. M., Gu, M. F., Rasmussen, A. \& Kaastra, J. 2003, ApJ, 587, 31

Vink, J. et al. 2001, ApJ, 560, L79

Willingale, R., Bleeker, J. A. M., van der Heyden, K. J., Kaastra, J. S. \& Vink, J. 2002, A\&A, 381,1039

Woods, P. \& Thompson, C. 2004, astro-ph/0406133

\section{Discussion}

UBertini: Do you think the hard x-ray continuum in the Cas A spectrum below the Ti line could be due by electron synchrotron emission at the shock front of the expanding SNR?

VINK: The continuum could be either non-thermal emission from $\sim 100 \mathrm{keV}$ electrons, accelerated by forward or internal shocks, or X-ray synchrotron from TeV electrons. In order to get synchrotron emission at $\sim 50 \mathrm{keV}$ you need very high $(>2000 \mathrm{Km} / \mathrm{s})$ shock velocities, which are likely to exist only at the forward shock. 